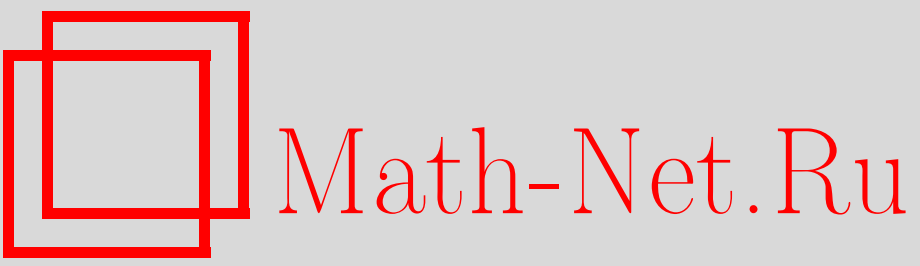

В. Р. Халилов, Заряженные векторные бозоны в магнитном поле при конечной температуре, ТМФ, 1998, том 114, номер 3, 439-453

DOI: https://doi.org/10.4213/tmf851

Использование Общероссийского математического портала Math-Net.Ru подразумевает, что вы прочитали и согласны с пользовательским соглашением

http://www.mathnet.ru/rus/agreement

Параметры загрузки:

IP : 54.80 .73 .141

26 апреля 2023 г., 13:56:08 
ТЕОРЕТИЧЕСКАЯ

И МАТЕМАТИЧЕСКАЯ

ФИЗИКА

Том 114, № 3

март, 1998

(C) $1998 \mathrm{r}$.

В.Р. Халилов*

\title{
ЗАРЯЖЕННЫЕ ВЕКТОРНЫЕ БОЗОНЫ В МАГНИТНОМ ПОЛЕ ПРИ КОНЕЧНОЙ ТЕМПЕРАТУРЕ
}

\begin{abstract}
Изучается поведение газа заряженных векторных бозонов конечной плотности во внешнем магнитном поле при конечной температуре. Бозе-эйнштейновской конденсации в присутствии однородного магнитного поля не происходит, хотя значительное количество бозонов будет находиться в основном состоянии. Показано, что в газе векторных бозонов может возникать спонтанная намагниченность, а также, как можно определить полную вероятность рождения пар векторных бозонов из вакуума сильным магнитным полем при конечной температуре. Получены выражения, которыми, по-видимому, можно охарактеризовать свободную энергию и магнитный момент вакуума, и, кроме того, выражения для плотности и магнитного момента рожденных полем пар векторных бозонов при конечной температуре.
\end{abstract}

\section{1. ВВЕДЕНИЕ}

Известно, что трехмерный идеальный газ нерелятивистских массивных скалярных частиц должен обнаруживать явление конденсации, предсказанное Бозе [1] и Эйнштейном [2], и эффект Мейсснера-Оксенфельда (МО) [3, 4] при температуре ниже чем так называемая критическая [5-7]. Внешнее однородное магнитное поле сушественно влияет на характер движения (квантование орбит в поперечном полю направлении) и спектр энергий (уровни Ландау) заряженных частиц. Впервые вопрос о бозе-эйнштейновской конденсации (БЭК) нерелятивистских скалярных заряженных бозонов исследовался в работе [3], автор которой пришел к выводу о невозможности БЭК скалярных заряженных частиц в присутствии даже слабого магнитного поля.

Известно, что в рамках квантово-полевого подхода сам эффект БЭК в релятивистском случае можно интерпретировать как явление спонтанного нарушения симметрии соответствуюшей эффективной квантово-полевой модели [8-10]. Таким образом, изучение БЭК представляет и самостоятельный интерес для квантовой теории поля. Нелишне также напомнить, что эффект Мейсснера рассматривается в качестве основного

* Московский государственный университет, Москва, Россия 
условия, которое позволяет дать микроскопическую интерпретацию сверхпроводимости при низких температурах.

Новый интерес к проблемам магнетизма и БЭК газов релятивистских заряженных частиц в сильных внешних магнитных полях при конечных температуре (достаточно высокой) и плотности в пространствах произвольной размерности возник в последние годы. В частности, в работе [11] было отмечено, что и в отсутствие БЭК релятивистский газ заряженных скалярных частиц должен проявлять эффект Мейсснера в области высоких температур и, следовательно, вести себя подобно "релятивистскому сверхпроводнику". Обшее доказательство невозможности БЭК для заряженных скалярных частиц в присутствии однородного магнитного поля в пространствах произвольной размерности дано Томсом [12].

Решение проблемы БЭК во внешнем магнитном поле необходимо как для развития теории, так и для экспериментальных приложений, тем более что в последнее время БЭК (в отсутствие внешнего поля) активно изучается экспериментально [13-15]. БЭК, по-видимому, впервые наблюдалась недавно при испарении атомов ${ }^{87} \mathrm{Rb}$ в магнитной ловушке, порождаюшей эллипсоидальный потенциал [13]. Особо следует подчеркнуть, что система не представляла собой изотропный идеальный газ, в частности, вследствие анизотропии ограничиваюшего потенциала продольные значения импульсов превышали поперечные, что порождает некоторые аналогии со случаем БЭК заряженных бозонов в слабом магнитном поле. Сушествуют две точки зрения, касаюшиеся самой проблемы БЭК в присутствии внешнего поля (см., например, [16]), а именно, как понимать БЭК: 1) как нахождение конечной части полного числа частиц в основном квантовом состоянии при некоторой ненулевой температуре; 2) как сушествование критической температуры, при которой химический потенциал становится равным нулю и ниже которой значительное количество частиц образует конденсат в основном квантовом состоянии.

Интерес к изучению поведения заряженных частиц с разными спинами в сильных магнитных полях при конечной температуре обусловливается в настояшее время открытием сверхсильных полей в нейтронных звездах $[17,18]$. Эта задача также представляет интерес для электрослабой модели Вайнберга-Салама, т. к. эта модель содержит заряженные векторные $W^{ \pm}$-бозоны, поведение которых в магнитном поле привлекает внимание исследователей, особенно в связи с возможностью появления в присутствии сверхсильного однородного магнитного поля так называемой нестабильной моды [19, 20]. Известно $[19,20]$, что спектр энергии заряженного векторного бозона массы $m$, заряда $е$ и спина $S=1$ в постоянном однородном магнитном поле, которое в декартовых координатах имеет вид $\mathbf{B}=(0,0, B)$, определяется формулой

$$
E_{n}(p)=\sqrt{m^{2}+(2 n+1-2 S) e B+p^{2}}, \quad S=-1,0,1,
$$

где квантовое число $n(n=0,1,2, \ldots)$ нумерует уровни Ландау, а $p$-проекция импульса на направление магнитного поля. Для $n=0, p=0, S=1$ величина $E$ обрашается в нуль при $B=B_{\mathrm{cr}} \equiv m^{2} / e$, а при $B>B_{\text {cr }}$ становится чисто мнимой. Такое поведение 
величины $E$ отражает квантовую неустойчивость поля заряженного векторного бозона в присутствии внешнего сильного однородного магнитного поля, которая обусловлена взаимодействием магнитного момента бозона с внешним однородным магнитным полем $[19,20]$. Соответствуюшая мода поля векторных бозонов называется нестабильной. Используя полный лагранжиан электрослабой модели Вайнберга-Салама, в котором также были учтены члены 4-го порядка, авторам работы [21] удалось при некоторых определенных условиях построить новые классические статические "магнитные" решения, описывающие $W$-бозонный конденсат в сильном магнитном поле в “древесном" приближении.

В данной статье будут рассмотрены магнитные свойства газа заряженных векторных бозонов в присутствии однородного магнитного поля при конечной температуре. Будет показано, что в газе векторных бозонов может возникать спонтанная намагниченность, а также оценена плотность образовавшихся пар за счет тепловых столкновений реальных частиц в плотной горячей среде во внешнем магнитном поле в области значений поля $B \simeq B_{\text {cr. }}$ В статье рассмотрены квантовые радиационные поправки в эффективный потенциал, учет которых необходим, особенно в том случае, когда конденсат пар векторных бозонов образуется из вакуума вследствие воздействия на последний сверхсильного магнитного поля при конечной температуре в области значений поля $B>B_{\mathrm{cr}}$. Кроме того, в этой работе делается попытка определить полную вероятность рождения пар векторных бозонов из вакуума сильным магнитным полем при конечной температуpe, а также получены выражения для свободной энергии, магнитного момента вакуума, плотности и магнитного момента пар (рожденных полем) векторных бозонов при конечной температуре. Частично эти задачи рассматривались в [16, 22-24].

В статье используется система единиц, в которой $c=\hbar=1$.

\section{2. ТЕРМОДИНАМИЧЕСКИЙ ПОТЕНЦИАЛ}

Термодинамический потенциал $\Omega$ газа векторных бозонов как функция химического потенциала $\mu$, индукции магнитного поля $B$ и температуры $T \equiv 1 / \beta$ определяется формулой

$$
\begin{aligned}
\Omega= & \frac{e B V}{4 \pi^{2} \beta}\left\{\int d p \ln \left[1-\exp \beta\left[\mu-\left(m^{2}-e B+p^{2}\right)^{1 / 2}\right]\right]+\right. \\
& \left.+\sum_{n=0}^{\infty} g_{n} \int d p \ln \left[1-\exp \beta\left[\mu-\left(m^{2}+(2 n+1) e B+p^{2}\right)^{1 / 2}\right]\right]\right\},
\end{aligned}
$$

где $V$ - объем, занимаемый газом, а $g_{n}=3-\delta_{0 n}-$ кратность вырождения возбужденных состояний. Произведя разложение логарифмов в $(2)$ и интегрируя по $p$, представим $\Omega$ в 
виде

$$
\begin{aligned}
\Omega(\mu) \equiv \Omega_{1} & +\Omega_{2}+\Omega_{3}=\frac{V e B}{2 \pi^{2} \beta}\left\{-M_{-} \sum_{k=1}^{\infty} k^{-1} \exp (k \beta \mu) K_{1}\left(k \beta M_{-}\right)+\right. \\
& +M_{+} \sum_{k=1}^{\infty} k^{-1} \exp (k \beta \mu) K_{1}\left(k \beta M_{+}\right)-3 \sum_{n=0}^{\infty} \sqrt{m^{2}+(2 n+1) e B} \times \\
& \left.\times \sum_{k=1}^{\infty} k^{-1} \exp (k \beta \mu) K_{1}\left(k \beta \sqrt{m^{2}+(2 n+1) e B}\right)\right\}
\end{aligned}
$$

где $M_{\mp} \equiv \sqrt{m^{2} \mp e B}$, а $K_{n}(x)$ - функция Макдональда порядка $n$.

Если газ нейтрален и состоит из частиц и античастиш, то в вьшеприведенных формулах необходимо $\exp (k \beta \mu)$ заменить на $2 \operatorname{ch}(k \beta \mu)$. Как известно $[5,6]$, термодинамический потенциал как функция химического потенциала является действительной функцией для действительных значений химического потенциала $\mu$, удовлетворяюших для частиц и античастиц с эффективной массой $M_{-}$условию

$$
|\mu| \leq M_{-} .
$$

Это условие вытекает из физического требования к числам заполнения частиц и античастиц с эффективной массой $M_{-}$быть положительно-определенными для любых действительных значений импульса $p$. Полная равновесная плотность частиц $\rho$ определяется формулой

$$
\rho=-\frac{1}{V} \frac{\partial \Omega}{\partial \mu}
$$

С помошью выражений (3) и (5) можно представить равновесную плотность бозонов в состоянии $n=0, S=1$ в виде

$$
\rho_{g}=\frac{e B M_{-}}{2 \pi^{2}} \sum_{k=1}^{\infty} K_{1}\left(k \beta M_{-}\right) \exp (k \beta \mu)
$$

Выражение для плотности (6) расходится, если химический потенциал $\mu$ стремится $\mathrm{K}$ его верхнему предельному значению $M_{-}$при низкой температуре $\left(\beta M_{-} \gg 1\right)$, что легко показать, используя асимптотическое представление $K_{1}(x)$ при больших значениях $x$. В этом пределе в выражении (6) рядпо $k$ имеет вид расходящегося ряда $\sum_{k=1}^{\infty} k^{-1 / 2}$. Этот результат ранее был получен в [22] методом преобразования Меллина. Расходимость в $\rho_{g}$ подразумевает, что значительное количество частиц может находиться в состоянии $n=0, S=1$ и иметь проекции импульсов на направление магнитного поля, отличные от нуля при любой конечной температуре. Поэтому БЭК невозможна в слабом однородном магнитном поле, по крайней мере, если мы интерпретируем БЭК во внешнем магнитном поле и без него одинаково (о другом подходе к проблеме см. [16]). 
Рассмотрим магнитные свойства газа векторных бозонов в слабом внешнем магнитном поле, т.е. когда индукция поля $B$ удовлетворяет неравенству $B \ll m^{2} / e$. Будем также предполагать, что $e B / m \ll T<m$. Последнее просто означает, что расстояние между уровнями Ландау много меньше средней тепловой энергии. При этих условиях вклады $\Omega_{1}$ и $\Omega_{2}$ в (3) малы по сравнению с вкладом $\Omega_{3}$, и поэтому $\Omega \approx \Omega_{3}$. В пределе относительно слабого внешнего магнитного поля $e B \ll m^{2}$ мы можем использовать для суммирования по $n$ в $\Omega_{3}$ формулу суммирования Эйлера

$$
\sum_{n=0}^{\infty} f(n+1 / 2)=\int_{0}^{\infty} f(x) d x+(1 / 24) f^{\prime}(0)
$$

и затем формулу [25]

$$
\int_{1}^{\infty} d z z^{2} K_{1}(k m z \beta)=\frac{1}{k m \beta} K_{2}(k m \beta) .
$$

В результате для $\Omega$ получаем представление

$$
\Omega=-\frac{3 V m^{2}}{2 \pi^{2} \beta^{2}} \sum_{k=1}^{\infty} \exp (k \beta \mu)\left[\frac{1}{k^{2}} K_{2}(k m \beta)-\frac{(e B \beta)^{2}}{24 m^{2}} K_{0}(k m \beta)\right] \text {. }
$$

Термодинамический потенциал (9) совпадает с термодинамическим потенциалом газа скалярных бозонов, умноженным на 3 [26, 27]. Из выражений (5) и (9) для равновесной плотности частиц в “возбужденных" состояниях в слабом поле можно получить, что

$$
\rho=\frac{3 m^{2} T}{2 \pi^{2}} \sum_{k=1}^{\infty} \exp (k \beta \mu)\left[\frac{1}{k} K_{2}(k m \beta)-\frac{(e B \beta)^{2}}{24 m^{2}} k \beta K_{0}(k m \beta)\right] .
$$

При низких температурах $T \ll m$ вдали от точки расходимости $\mu=M_{-}$плотности бозонов в основном состоянии (6) выражения для термодинамического потенциала (3) и полной плотности бозонов нетрудно привести к известному в литературе виду

$$
\begin{aligned}
\Omega & \simeq-\frac{V T^{1 / 2} m^{3 / 2}}{(2 \pi)^{3 / 2}}\left[3 T^{2} \operatorname{Li}_{5 / 2}\left(e^{\beta(\mu-m)}\right)+\frac{7(e B)^{2}}{8 m^{1 / 2}} e^{\beta(\mu-m)}\right], \\
\rho & \simeq 3\left(\frac{T m}{2 \pi}\right)^{3 / 2} \operatorname{Li}_{3 / 2}(1)\left[1-\frac{7(e B \beta)^{2}}{2^{1 / 2} 96 \pi m^{2}}\right],
\end{aligned}
$$

где $\operatorname{Li}_{n}(x)=\sum_{k=1}^{\infty} x^{k} / k^{n}$ - полилогарифмическая функция порядка $n$. Магнитный момент газа при тех же предположениях есть

$$
M_{z}(B)=\frac{7 e^{2} B T^{1 / 2}}{4 m^{1 / 2}(2 \pi)^{3 / 2}} e^{\beta(\mu-m)} .
$$

Видно, что магнитный момент - положительная функция внешнего поля и температуры газа, так что парамагнитный (спиновый) вклад доминирует. 


\section{3. ПРЕДЕЛ СИЛЬНОГО ПОЛЯ, НАМАГНИЧЕННОСТЬ}

Изучим теперь магнитные свойства газа векторных бозонов заданной плотности в

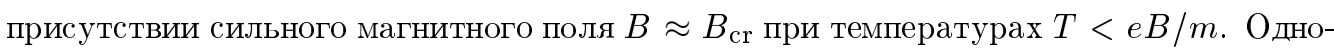
родное внешнее магнитное поле имеет особенность собирать все квантовые состояния, лежашие в интервале энергии $\mathrm{eB} / \mathrm{m}$. Переходы бозонов с уровня Ландау $n=0$ на возбужденные уровни $n \geq 1$ не будут разрешены, если $T<e B / m$ и все бозоны в квантовом состоянии с $n=0$ могут быть рассмотрены как конденсат в двумерном "импульсном" пространстве в плоскости, перпендикулярной направлению магнитного поля с "эффективными" импульсами $k<(e B)^{1 / 2}$. Реальный конденсат в трехмерном импульсном пространстве не образуется, поскольку продольные импульсы бозонов могут иметь значения вне этого интервала.

При низких температурах $\beta M_{-} \gg 1$ вклады в термодинамический потенциал (3) всех возбужденных состояний экспоненциально малы по сравнению с вкладом состояния с $n=0$. Поэтому достаточно рассмотреть только первое слагаемое $\Omega_{1}$ в (3) в этом пределе, т. е.

$$
\Omega(\mu)=-\frac{V e B M_{-}}{2 \pi^{2} \beta} \sum_{k=1}^{\infty} k^{-1} \exp (k \beta \mu) K_{1}\left(k \beta M_{-}\right) .
$$

Формула для плотности частиц, которую можно получить с помошью (14), совпадает c (6). В сильном магнитном поле выражение (6) расходится в пределе $\mu \rightarrow M_{-}$. Действительно, при $M_{-} \gg T, M_{-}-\mu<T$ из (6) следует, что

$$
\rho \cong \frac{e B\left(T M_{-}\right)^{1 / 2}}{(2 \pi)^{3 / 2}}\left[\left(\frac{\pi T}{M_{-}-\mu}\right)^{1 / 2}-1,46\right] .
$$

Отметим, что первый (основной) член в (15) совпадает с полученным в работе [16]. Вдали от точки конденсации свободного газа векторных бозонов, т. е. при относительно "высоких" температурах, в пределе $M_{-}<T$, но $m \gg T$ выражение для плотности газа векторных бозонов (6) приобретает вид

$$
\rho \cong \frac{e B T(1+\beta \mu)}{2 \pi^{2}} .
$$

Из формул (15) или (16) следует, что значительное количество частиц может находиться в состояниях с проекциями импульсов на направление магнитного поля, отличными от нуля. И так как теперь эти состояния следует рассматривать как возбужденные, то, следовательно, плотность бозонов, которые будут находиться вне основного состояния (c $p=0)$, будет отлична от нуля при любой ненулевой температуре; это означает, что БЭК (фактически в одномерном импульсном пространстве) невозможна в присутствии достаточно сильного однородного магнитного поля. Заметим, что на невозможность БЭК классического одномерного газа, по-видимому, впервые указал Фейнман [28]. 
Рассмотрим теперь намагниченность, производимую заряженными векторными бозонами в низшем энергетическом состоянии. Магнитная индукция $B$ определяется как

$$
B=H+4 \pi M(B)
$$

где $H$ - напряженность внешнего магнитного поля, а $M(B)$ - магнитный момент газа. Из выражения (14) получим

$$
M_{z}(B)=-\frac{1}{V} \frac{\partial \Omega}{\partial B}=\frac{e}{2 \pi^{2} \beta} \sum_{k=1}^{\infty}\left[\frac{M_{-}}{k} K_{1}\left(k \beta M_{-}\right)+\frac{e B \beta}{2} K_{0}\left(k \beta M_{-}\right)\right] \exp (k \beta \mu)
$$

Это точное выражение для магнитного момента газа векторных бозонов в низшем энергетическом состоянии в однородном магнитном поле. Видно, что магнитный момент (18) как функция индукции поля и температуры всегда положителен.

Если $\beta \gg 1$ и $\beta M_{-}>1$, то в (18) достаточно учесть только слагаемые с $K_{0}$. Используя асимптотические разложения для $K_{0}$ в $(18)$ и для $K_{1}$ в $(6)$, находим (см. также [16])

$$
\begin{gathered}
M_{z}(B)=\frac{e}{2 M_{-}} \rho \\
\rho=\frac{e B M_{-}^{1 / 2} T^{1 / 2}}{(2 \pi)^{3 / 2}} \operatorname{Li}_{1 / 2}\left(\exp \left[\beta\left(\mu-M_{-}\right)\right]\right) .
\end{gathered}
$$

Отметим, что последние формулы мы можем также получить, используя первый член следуюшего выражения для $\Omega$, справедливого при низких температурах:

$$
\begin{aligned}
\Omega= & -\frac{V e B M_{-}^{1 / 2}}{(2 \pi)^{3 / 2} \beta^{3 / 2}}\left[\operatorname{Li}_{3 / 2}\left(\exp \left[\beta\left(\mu-M_{-}\right)\right]\right)+\right. \\
& \left.+\frac{3}{8 \beta M_{-}} \operatorname{Li}_{5 / 2}\left(\exp \left[\beta\left(\mu-M_{-}\right)\right]\right)+\cdots\right] .
\end{aligned}
$$

Предел низких температур соответствует нерелятивистскому приближению. При относительно "высоких" температурах $T>M_{-}$магнитный момент газа также, разумеется, положителен:

$$
M_{z}(B) \simeq-\frac{e}{2 \pi^{2}}\left[e B \ln \left(M_{-} / 2 T\right)-T^{2}\right] .
$$

Обсудим теперь вопрос о возможности возникновения спонтанной намагниченности в рассматриваемой нами системе. Для этого нужно показать, что сушествует решение уравнения (17) при $H=0: H=0=B-4 \pi M(B)$. Из выражений (19) и (20) видно, что такое решение возможно только при низких температурах. Подставляя (19) в (17), при $H=0$ находим, что решение сушествует при плотности

$$
\rho=\frac{e B M_{-}}{2 \pi e^{2}}, \quad e B \approx m^{2}
$$


Следовательно, газ векторных бозонов в низшем энергетическом состоянии в магнитном поле при низкой температуре ферромагнитен [16]. Эта спонтанная намагниченность указывает нам на новьй возможный механизм возникновения сильных магнитных полей. Отметим, что уравнение (17) не имеет решений при $H=0$ в пределе "высоких" температур.

Оценим плотность пар векторных бозонов, рожденных (в низшем энергетическом состоянии) за счет столкновений заряженных бозонов в магнитном поле с $B \approx B_{\mathrm{cr}}$. В предположении, что плотность рожденных пар много больше плотности начальных частиц, для оценки можно использовать формулу (6) с $\mu=0$ [5]. Тогда для низких $\left(\beta M_{-}>1\right.$, но $T \ll m)$ и "высоких" $\left(\beta M_{-}<1, T<m\right)$ температур мы получим соответственно

$$
\begin{aligned}
& \rho_{T} \cong \frac{e B\left(M_{-} T\right)^{1 / 2}}{(2 \pi)^{3 / 2}} \exp \left(-\beta M_{-}\right), \\
& \rho_{T} \cong \frac{e B T}{2 \pi^{2}} .
\end{aligned}
$$

\section{4. ЭНЕРГИЯ ВАКУУМА И ПОЛНАЯ ВЕРОЯТНОСТЬ РАСПАДА}

Обсудим вопрос о связи термодинамического потенциала как физической величины, характеризующей газ реальных частиц, и эффективного потенциала, которьй представляет собой величину, рассматриваемую в квантовой теории поля [29]. В однопетлевом приближении эффективный потенциал должен содержать две части (не зависяшую и зависяшую от температуры). В присутствии внешнего однородного магнитного поля его можно представить в виде (см., например, [30])

$$
V=V_{0}+\Omega(0, B, \beta)
$$

причем в $\Omega$ мы должны положить $\mu=0$. Отметим, что формула (26) и все величины ниже относятся к единице объема.

Видно, что радиационные поправки определяются спектром энергии заряженной частицы во внешнем поле и зависят от температуры. Первый член формулы (26) описывает вклад в эффективный потенциал в единице объема, не зависящий от температуры, второй - совпадает с термодинамическим потенциалом идеального газа бозонов, в котором химический потенциал положен равным нулю. Следовательно, вычисление зависящей от температуры части эффективного потенциала может быть сведено к вычислению термодинамичесого потенциала при $\mu=0$. Эффективный потенциал (26) является основной величиной, которая изучается при исследовании свойств вакуума квантово-полевой модели во внешнем поле при конечной температуре. Нелишне напомнить, что теперь нас интересуют эффекты, обусловленные внешним магнитным полем при конечной температуре, а не плотностью реальных бозонов.

Известно $[19,20,24]$, что выражение для эффективного потенциала при $T=0$ во внешнем однородном магнитном поле с $B>B_{\text {cr }}$ становится комплексным, что обусловлено вкладом нестабильной бозонной моды с эффективной массой $M_{-}$. Мнимая часть 
эффективного потенциала появляется вследствие того, что “спектр энергии” заряженного массивного векторного бозона становится мнимым при $B>B_{\mathrm{cr}}$. Из формулы (14) следует, что часть эффективного потенциала, зависяшая от температуры, также ста-

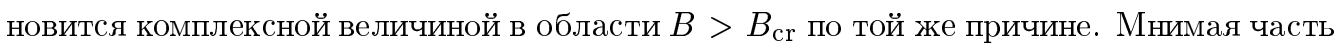
эффективного потенциала могла бы не появиться вообще, если бы при вычислениях мы учитывали только вклад “физической” части спектра энергий, которую можно определить лишь в области $B<B_{\text {cr. }}$. Однако, по-видимому, будет более правильным учесть весь "спектр энергий”, но при этом нужно попытаться дать интерпретацию получающихся комлексных выражений для квантовых поправок. В частности, возникает вопрос об интерпретации мнимой части эффективного потенциала , а также других величин, которыми можно описать изучаемую квантовую статистическую систему.

Сформулированный вопрос можно попытаться проанализировать, как это делается в стандартной нерелятивистской квантовой механике при исследовании квазистационарных состояний. Действительно, известно [31], что временная зависимость волновой функции нестабильной нерелятивистской квантово-механической системы имеет вид

$$
\Psi \approx \exp (-i E t)=\exp -t(i \Re E+\Im E),
$$

где $\Im E$ определяет "ширину" уровня энергии и полную вероятность распада квантовой системы

$$
w=-2 \Im E .
$$

В рассматриваемом нами случае аналогом нестабильной квантовой системы является вакуум заряженных векторных бозонов во внешнем магнитном поле при конечной температуре.

Следуя этой аналогии, чтобы найти полную вероятность распада вакуума, мы должны вычислить энергию вакуума, используя формулы статистической физики, т. к. волновая функция вакуума с учетом рождения пар должна описывать многочастичное состояние. Часть энергии, зависящую от температуры, найдем по формуле [5]

$$
E(T, B)=\Omega_{0}-T \frac{\partial \Omega_{0}}{\partial T} .
$$

Мнимая часть $V_{0}$ хорошо известна (см., например, [24]):

$$
\Im V_{0}=-\frac{e B\left(e B-m^{2}\right)}{8 \pi} .
$$

Полная вероятность физического процесса, разумеется, должна быть положительной величиной. Это требование позволяет однозначно выполнить аналитическое продолжение в область $B>B_{\mathrm{cr}}$, в которой эффективный потенциал и “энергия вакуума" имеют комплексные значения. 


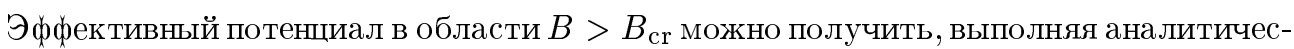
кое продолжение функции $K_{1}(z)$, рассматриваемой как функция переменной $z=k \beta M_{-}$, в комлексную плоскость. Для этого определим аргумент функции $K_{1}(z)$ в области $B>B_{\text {cr }}$ следующим образом:

$$
z= \pm i k \beta M_{-} \equiv \pm i t
$$

Тогда согласно принципу симметрии Шварца имеем

$$
K_{1}( \pm i t)=K_{1}^{*}(\mp i t)=-\frac{\pi}{2}\left[J_{1}(t) \mp i N_{1}(t)\right]
$$

где $J_{1}(t)$ и $N_{1}(t)$ - цилиндрические функции первого и второго родов, т. е. функции Бесселя и Неймана, соответственно. При выполнении аналитического продолжения в область $B>B_{\text {сr }}$ следует выбрать такие знаки перед аргументом $i t$ в $K_{1}( \pm i t)$ и перед $M_{-}$в (14), чтобы полная вероятность была положительной и сводилась к случаю нулевой температуры. В результате для $\Omega_{0}(t)$ получим выражение

$$
\Omega_{0}(t)=\frac{e B T \sqrt{e B-m^{2}}}{2 \pi} \sum_{k=1}^{\infty} \frac{1}{k}\left[i J_{1}(t)+N_{1}(t)\right]
$$

Ряд, описывающий мнимую часть $\Omega_{0}(t)$, удобно преобразовать в сумму с помошью следуюшей формулы:

$$
\sum_{k=1}^{\infty} \frac{J_{1}(k t)}{k}=1-\frac{t}{4}+\frac{2}{t} \sum_{n=1}^{l} \sqrt{t^{2}-(2 \pi n)^{2}}, \quad 2 \pi l<t<2 \pi(l+1) .
$$

Подставляя мнимую часть $\Omega_{0}(t)$ в $(29)$, найдем член $\Im E(T)$, зависящий от температуры. Затем, взяв сумму величины (30) и найденного члена, с учетом согласования знаков, мы получим выражение для полной вероятности распада вакуума в виде

$$
w=\frac{e B\left(e B-m^{2}\right)}{8 \pi}\left[1+\frac{8 T^{2}}{e B-m^{2}} \sum_{n=1}^{l} \frac{2 \pi n^{2}}{\sqrt{x^{2}-n^{2}}}\right]
$$

где $l$ - целая часть функции $\left[x=\beta \sqrt{e B-m^{2}} / 2 \pi\right]$ (наибольшее целое число меньше $x$ ).

Выражение (35) можно также интерпретировать как полную вероятность рождения пар векторных бозонов (в низшем квантовом состоянии) внешним магнитным полем из вакуума при конечной температуре, причем первый член этого выражения определяет полную вероятность указанного процесса при нулевой температуре. Видно, что полная вероятность, определенная формулой (35), всегда положительна и сводится к соответствующему выражению того же процесса при $T=0$. Процесс рождения пар векторных бозонов из вакуума внешним магнитным полем имеет порог по величине напряженности магнитного поля $\left(B>B_{\mathrm{cr}}\right)$. 


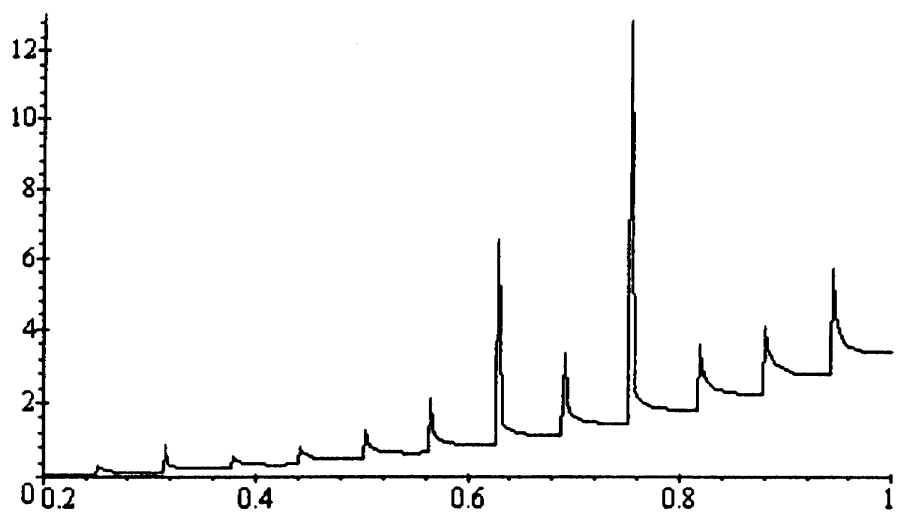

Рис 1.

Отметим также, что полная вероятность рождения пар векторных бозонов (35) при $T \neq 0$ больше, чем вероятность при $T=0$, т. к. в присутствии термостата появляются новые вакуумные состояния, которые могут заполняться рожденными полем бозонами.

Полная вероятность рождения пар в зависимости от напряженности магнитного поля при низких температурах приведена на рис. 1. По оси ординат отложена функция $8 \pi w(\beta m, x)(10 / m)^{4}$ в зависимости от $x=\left(B / B_{\mathrm{cr}}\right)-1$ при $\beta m=100$. Острые пики на рис. 1 в узких областях значений поля соответствуют случаям, когда пары векторных бозонов рождаются точно на дискретных "квантовых уровнях, зависящих от температуры", т.е. в новых квантовых состояниях, которые появляются в термостате [32].

При определении полной вероятности рождения пар векторных бозонов мы учли мнимую часть эффективного потенциала в однопетлевом приближении при $T=0$, которая появляется, если рассмотренный выше процесс разрешен. Реальную часть эффективного потенциала с учетом однопетлевого вклада заряженных векторных бозонов при $T=0$ можно получить в простом виде только в пределе сверхсильного магнитного поля $e H \gg m^{2}[24]$ :

$$
\Re V_{0} \approx \frac{7(e H)^{2}}{32 \pi^{2}}\left[\ln \left(\frac{e H}{m^{2}}\right)-0.9\right],
$$

здесь $e H$ - произведение заряда на напряженность внешнего магнитного поля (ренорминвариантная величина), а $m$ имеет смысл перенормированной массы бозона.

Если вакуумное состояние W-бозонного сектора электрослабой модели Вайнберга-Салама во внешнем магнитном поле при конечной температуре рассматривать как некоторую квантово-статистическую систему, то аналогом свободной энергии этой системы, по-видимому, является термодинамический потенциал (33).

При относительно низких температурах, когда $\beta \sqrt{e B-m^{2}} \gg 1$, реальная и мнимая части свободной энергии вакуума (33) как функции внешнего магнитного поля осциллируют с увеличиваюшейся амплитудой при увеличении напряженности поля и имеют

5 Теоретическая и математическая физика, т. 114, № 3, 1998 г. 


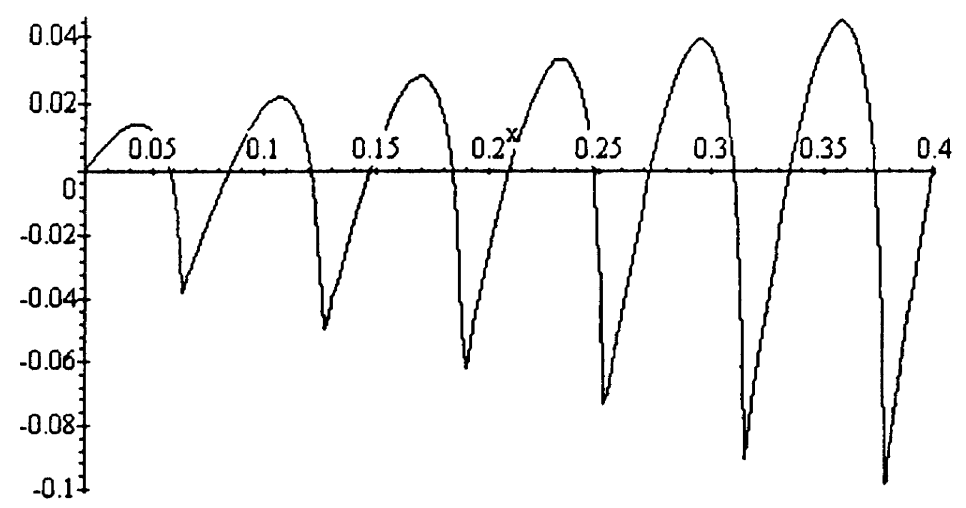

Рис 2.

минимумы и нули, причем минимумы реальной и нули мнимой частей свободной энергии достигаются при одних и тех же значениях напряженности поля. Такое поведение свободной энергии следует из поведения функций $J_{1}(t)$ и $N_{1}(t)$ [25], которые осциллируют с одинаковыми амплитудами, но сдвинуты по фазе на $\pi / 2$, по крайней мере, при больших значениях аргумента, т.е. при низких температурах. Строго говоря, свободная энергия (так же как и энергия) любой физической модели должна быть действительной величиной. Тем не менее иногда мы употребляем фразу “мнимая часть свободной энергии”, чтобы просто подчеркнуть, что математическое выражение этой физической величины в некоторой области параметров содержит как действительную, так и мнимую части.

Поведение свободной энергии модели Вайнберга-Салама изучалось численными методами в области значений внешнего магнитного поля $B_{\mathrm{cr}}<B<2 B_{\text {cr }}$ и температуры $m \gg T$. Для примера мы приведем лишь график зависимости функции $4 \pi \Re \Omega_{0} / m^{4}$ от $x=\sqrt{(B / B)_{\text {cr }}-1}$ при $\beta m=100$ (рис. 2), который хорошо иллюстрирует обсуждаемую осциллящионную зависимость реальной и мнимой частей свободной энергии от напряженности магнитного поля при относительно низкой температуре.

Нули мнимой части свободной энергии соответствуют значениям поля (при заданной температуре), при которых изучаемая квантовая модель стабильна в смысле термодинамики. Глубокие и острые минимумы реальной части свободной энергии как функции $B$ появляются, поскольку "квантовые уровни, зависящие от температуры", которые могут заполняться рожденными из вакуума бозонами, являются дискретными. Реальную часть свободной энергии можно также интерпретировать как функцию, зависяшую от переменной термодинамического состояния (которая в нашем случае есть количество рожденных бозонных пар) при заданных температуре и объеме. Отметим, что осциллируюшее поведение термодинамического потенциала в однопетлевом приближении для чистой $\mathrm{SU}(2)$-калибровочной модели в присутствии однородного магнитного поля было получено в работе [33]. 


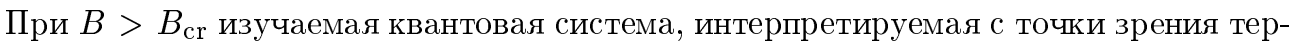
модинамики, является неустойчивой. При этом рожденные полем пары частиц будут находиться в состоянии термодинамического равновесия при заданных температуре и объеме только в том случае, если свободная энергия термодинамической системы (т.е. реальная часть (33)) имеет минимумы. Следует подчеркнуть, что неустойчивое равновесное состояние вообще не может существовать в термодинамике [34], поэтому физический смысл имеют только точки, в которых реальная и мнимая части (33) имеют минимумы и нули, соответственно. Статистическая система будет устойчивой только в том случае, если мнимая часть свободной энергии точно равна нулю. Поскольку пики полной вероятности распада вакуума, минимумы реальной и нули мнимой (зависяшей от температуры) частей свободной энергии достигаются при одних и тех же значениях внешнего поля (при заданной температуре), можно сказать, что вакуум модели Вайнберга-Салама стабилен только при этих значениях напряженности поля.

При относительно высоких температурах $\beta \sqrt{e B-m^{2}} \sim 1$ полная вероятность рождения пар векторных бозонов определяется только первым членом правой части формулы (35), а значения температуры и величины внешнего поля теперь удовлетворяют неравенству

$$
0<\sqrt{e B-m^{2}}<2 \pi T
$$

Интересно, что мнимая (зависящая от температуры) часть свободной энергии обрашается в нуль при $\beta \sqrt{e B-m^{2}}=4$. Нетрудно видеть, что поведение всех рассматриваемых величин при “высокой” температуре существенно отличается от случая низких температур: они не обнаруживают ни осцилляций, ни пиков.

\section{5. ЗАКЛЮЧЕНИЕ}

Выполняя вычисления, мы считали, что магнитное поле постоянно во времени. Однако, если пары спонтанно рождаются из вакуума исключительно за счет магнитного поля (аналогично механизму Швингера для рождения электрон-позитронных пар в присутствии электрического поля [35]), внешнее магнитное поле будет изменяться со временем. Можно, однако, считать, что оно будет оставаться постоянным в течение промежутка характеристического времени, равного приблизительно $1 /\left|M_{-}\right|$, поэтому все полученные выше физические величины следует отнести к этому промежутку времени, т.е. умножить на $1 /\left|M_{-}\right|$. Так, плотность рожденных полем пар векторных бозонов $\rho_{v}$ и их магнитный момент $M$ можно определить как $\rho_{v}=w /\left|M_{-}\right|$и $M=e \rho_{v} / m$. Отсюда видно, что плотность рожденных полем бозонов приблизительно в $4 \pi / e^{2}$ раз меньше плотности, которая необходима, чтобы индукция магнитного поля, возникающая в результате спонтанной намагниченности бозонов, достигла значения $B_{\text {cr }}$ (cм. (23)).

Следует подчеркнуть, что плотность пар векторных бозонов, рожденных (в низшем энергетическом состоянии) в результате столкновений бозонов в магнитном поле с $B \approx$ $B_{\mathrm{cr}}$, определяется формулами $(24)$, если $\beta\left|M_{-}\right|>1, T \ll m$, и $(25)$, если $\beta\left|M_{-}\right|<1$, $T<m$. 
Отметим, что изучение различных эффектов в сильных магнитных полях представляет не только академический интерес. Выше уже упоминалось о сушествовании сильных магнитных полей в нейтронных звездах. В работах $[36,37]$ указывается на возможность появления очень сильных (правда, неоднородных) магнитных полей вблизи точки электрослабого фазового перехода. Если газ заряженных частиц конечной плотности находится в сверхсильном магнитном поле, становится актуальной задача самого определения термодинамических величин (например, свободной энергии и магнитного момента), которыми следует характеризовать такую систему. Дело в том, что в сильном магнитном поле вклад вакуума (т.е. виртуальных заряженных частиц) во все физические величины, описывающие систему частиш конечной плотности, может превышать вклад частиц [38]. Неясно также, должна ли быть свободная энергия вакуума функцией индукции или напряженности магнитного поля. Мы надеемся обсудить эти вопросы более подробно отдельно.

В заключение мне приятно поблагодарить Чун Лин Хо, Ян Ши и В. Н. Родионова за стимулирующие и полезные дискуссии.

\section{Список литературы}

[1] S. N. Bose. Z. Phys. 1921. B. 26. S. 178.

[2] A. Einstein. Sitzungsber. Preuss. Akad. Wiss. 1924. B. 22. S. 261.

[3] M.R. Schafroth. Phys. Rev. 1955. V. 100. P. 463.

[4] R. M. May. Phys. Rev. 1959. V. 115. P. 254; J. Math. Phys. 1965. V. 6. P. 1446.

[5] Л. Д. Ландау, Е. М. Лифшич. Статистическая физика. Часть 1. М.: Наука, 1976.

[6] А. Исихара. Статистическая физика. М.: Мир, 1973.

[7] Р. Кубо. Статистическая механика. М.: Мир, 1967.

[8] H. E. Haber, H. A. Weldon. Phys. Rev. D. 1982. V. 25. P. 502.

[9] J. Kapusta. Phys. Rev. D. 1981. V. 24. P. 426.

[10] D. J. Toms. Phys. Rev. D. 1995. V. 51. P. 1886, 1895.

[11] J. Daicic, N. E. Frankel, V. Kowalenko. Phys. Rev. Lett. 1993. V. 71. P. 1779.

[12] D. J. Toms. Phys. Lett. B. 1995. V. 343. P. 259.

[13] M. H. Anderson et al. Science. 1995. V. 269. P. 198.

[14] K. B. Davis. Phys. Rev. Lett. 1995. V. 75. P. 3969.

[15] C. C. Bradley et al. Phys. Rev. Lett. 1995. V. 75. P. 1687.

[16] H. P. Rojas. Phys. Lett. B. 1996. V. 179. P. 148.

[17] J. Trümper, W. Pietsch, C. Reppin et al. Astrophys. J. 1978. V. 219. P. L105.

[18] W. A. Wheaton, J. P. Doty, F. A. Primini et al. Nature. 1979. V. 282. P. 240.

[19] В. В. Скалозуб. ЯФ. 1978. Т. 28. С. 228.

[20] N. K. Nielsen, P. Olesen. Nucl. Phys. B. 1979. V. 144. P. 376.

[21] J. Ambjørn, P. Olesen. Phys. Lett. B. 1989. V. 218. P. 67; Nucl. Phys. B. 1990. V. 330. P. 193.

[22] J. Daicic, N. E. Frankel. Phys. Rev. D. 1995. V. 52. P. 7174.

[23] А.С. Виивцев, В. Ч. Жуковский, А. О. Старинец. Препринт 44/91. Томск: Томский научный центр СОРАН, 1991.

[24] V. R. Khalilov. Electrons in Strong Electromagnetic Fields: an Advanced Classical and Quantum Treatment. Amsterdam: Gordon \& Breach Sci. Pub., 1996. 
[25] И. С. Градштейн, И. М. Рыжсик. Таблицы интегралов, сумм, рядов и произведений. М.: ГИФМЛ, 1963.

[26] D. E. Miller, P. S. Ray. Phys. Rev. A. 1986. V. 33. P. 1990.

[27] P. Elmfors, P. Liljenberg, D. Persson, Bo-S. Scagerstam. Phys. Lett. B. 1995. V. 348. P. 462.

[28] P. Фейнман. Статистическая механика. М.: Мир, 1975.

[29] K. Huang. Quarks Leptons and Gauge Fields. Singapore: World Scientific, 1982.

[30] J. Chakrabarti. Phys. Rev. D. 1981. V. 24. P. 2232.

[31] Л. Д. Ландау, Е. М. Лифииц. Квантовая механика. Нерелятивистская теория. М.: Наука, 1989.

[32] R.D. Mattuck. A Guide to Feynman Diagrams in the Many-body Problem. London: McGRAW-HILL Pub. Company, 1967.

[33] A. O. Starinets, A.S. Vshivtsev, V. Ch. Zhukovskii. Phys. Lett. B. 1994. V. 322. P. 403.

[34] Р. Кубо. Термодинамика. М.: Мир, 1970.

[35] J. Schwinger. Phys. Rev. 1951. V. 82. P. 664.

[36] T. Vachaspati. Phys. Lett. B. 1991. V. 265. P. 258.

[37] J. Ambjørn, P. Olesen. Niels Bohr Institute preprint NBI-HE-93-17, 1993.

[38] P. Elmfors, P. Liljenberg, D. Persson, Bo-S. Skagerstam. Phys. Rev. D. 1995. V. 51. P. 5885.

Поступила в редакцию 3.VII.1997 г. 